Multi-scale algorithm for improved scintillation detection in a CCD-based gamma camera

This article has been downloaded from IOPscience. Please scroll down to see the full text article.

2009 Phys. Med. Biol. 54831

(http://iopscience.iop.org/0031-9155/54/4/001)

View the table of contents for this issue, or go to the journal homepage for more

Download details:

IP Address: 131.180.130.114

The article was downloaded on 21/12/2010 at 10:49

Please note that terms and conditions apply. 


\title{
Multi-scale algorithm for improved scintillation detection in a CCD-based gamma camera
}

\author{
Marc A N Korevaar ${ }^{1,2}$, Jan W T Heemskerk ${ }^{1,2}$, Marlies C Goorden ${ }^{1,2}$ \\ and Freek J Beekman ${ }^{1,2,3}$ \\ ${ }^{1}$ Department of Nuclear Medicine, Image Sciences Institute, University Medical Center Utrecht, \\ Heidelberglaan 100, 3584 CG, Utrecht, The Netherlands \\ 2 Section of Radiation Detection and Matter, Department of R3, Applied Sciences, \\ Delft University of Technology, Mekelweg 15, 2629 JB, Delft, The Netherlands \\ ${ }^{3}$ Molecular Imaging Labs (MILABS), Universiteitsweg 100, 3584 CG, Utrecht, \\ The Netherlands \\ E-mail: m.a.n.korevaar@tudelft.nl
}

Received 18 July 2008, in final form 8 December 2008

Published 14 January 2009

Online at stacks.iop.org/PMB/54/831

\begin{abstract}
Gamma cameras based on charge-coupled devices (CCDs) and micro-columnar CsI scintillators can reach high spatial resolutions. However, the gamma interaction probability of these scintillators is low (typically $<30 \%$ at $141 \mathrm{keV}$ ) due to the limited thickness of presently available micro-columnar scintillators. Continuous scintillators can improve the interaction probability but suffer from increased light spread compared to columnar scintillators. In addition, for both types of scintillators, gamma photons incident at an oblique angle reduce the spatial resolution due to the variable depth of interaction (DOI). To improve the spatial resolution and spectral characteristics of these detectors, we have developed a fast analytic scintillation detection algorithm that makes use of a depth-dependent light spread model and as a result is able to estimate the DOI in the scintillator. This algorithm, performing multi-scale frame analysis, was tested for an electron multiplying CCD (EM-CCD) optically coupled to $\mathrm{CsI}(\mathrm{Tl})$ scintillators of different thicknesses. For the thickest scintillator (2.6 mm) a spatial resolution of $148 \mu \mathrm{m}$ full width half maximum (FWHM) was obtained with an energy resolution of $46 \%$ FWHM for perpendicularly incident gamma photons (interaction probability $61 \%$ at $141 \mathrm{keV}$ ). The multiscale algorithm improves the spatial resolution up to $11 \%$, the energy resolution up to $36 \%$ and the signal-to-background counts ratio up to $46 \%$ compared to a previously implemented algorithm that did not model the depth-dependent light spread. In addition, the multi-scale algorithm can accurately estimate DOI. As a result, degradation of the spatial resolution due to the variable DOI for gamma photons incident at a $45^{\circ}$ angle was improved from $2.0 \cdot 10^{3}$ to
\end{abstract}


$448 \mu \mathrm{m}$ FWHM. We conclude that the multi-scale algorithm significantly improves CCD-based gamma cameras as can be applied in future SPECT systems.

(Some figures in this article are in colour only in the electronic version)

\section{Introduction}

Today, small animal single-photon emission tomography (SPECT) pinhole imaging (Meikle et al 2005, Beekman and van der Have 2007) can yield excellent image resolutions (Jaszczak et al 1994, Schramm et al 2000, Liu et al 2004) down to the sub-half-mm scale (Beekman et al 2005, Vastenhouw et al 2007). At present these ultra-high resolutions are obtained using traditional gamma cameras, by employing the principle of pinhole magnification. For future improvements of small animal SPECT imaging, gamma cameras with better spatial resolution and significant energy discrimination are essential (Rogulski et al 1993, Barber 1999, Beekman \& Vastenhouw 2004, Rentmeester et al 2007). Compact, high-resolution gamma cameras using EM-CCDs in combination with micro-columnar CsI(Tl) scintillators (Nagarkar et al 1998) are being developed by many research groups (de Vree et al 2005, Nagarkar et al 2006, Meng 2006, Miller et al 2006, Heemskerk et al 2007). In such CCD-based detectors individual scintillation events can be detected in 'photon counting' mode, enabled by read-out at high frame rates. This detection method greatly improves the spatial resolution compared to integration of the scintillation light signal (Beekman and de Vree 2005). However, the presently applied micro-columnar scintillators suffer from low interaction probability for gamma photons $(<30 \%$ at $141 \mathrm{keV})$ due to the limited thickness of commercially available scintillators (typically $<1 \mathrm{~mm}$ ).

A solution to the latter problem is the use of continuous scintillators, which are available in larger thicknesses and are also more cost effective. Furthermore, continuous scintillators can be obtained for scintillator materials with higher atomic number $Z$ and density than $\mathrm{CsI}(\mathrm{Tl})$, offering potential for a further improvement in sensitivity. However, these scintillators have increased scintillation light spread and increased dependence of the light spread on the depth of interaction (DOI). This severely complicates accurate scintillation detection.

Gamma cameras equipped with both columnar and continuous scintillators suffer from degradation of spatial resolution due to the variable DOI for gamma photons incident at an oblique angle (figure 2(a)). Elsewhere a statistical scintillation detection algorithm, that uses information of the depth-dependent light spread, is applied to an EM-CCD-based gamma camera with a micro-columnar scintillator (Miller et al 2006). However, long computation times of statistical algorithms can be prohibitive in many applications. To overcome this problem, a fast analytical scintillation detection algorithm, employing an analytical model for the depth-dependent light spread, is presented and applied to a gamma camera with continuous scintillators. This analytical multi-scale algorithm (MSA) enables an accurate estimate of the DOI and is compared with a previously proposed detection algorithm based on a simple Gaussian filter algorithm (GFA) (de Vree et al 2005). The spatial resolution, energy resolution and the signal-to-background counts ratio (SBR) are evaluated for both algorithms using $\mathrm{CsI}(\mathrm{Tl})$ continuous scintillators of different thicknesses. 


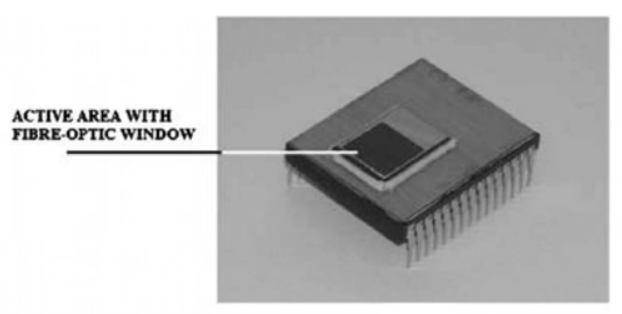

(a)

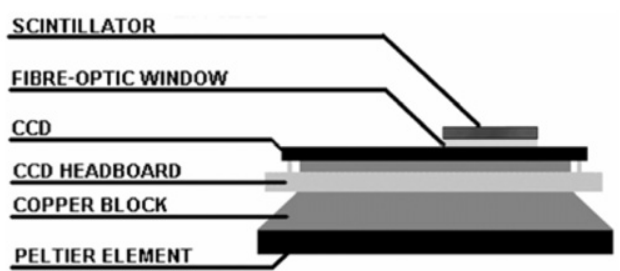

(b)

Figure 1. (a) EM-CCD with the fiber-optic window. (b) Schematic of the gamma camera consisting of a continuous scintillator optically coupled through a fiber-optic window to the EM-CCD, cooled by a Peltier element.

\section{Methods}

\subsection{EM-CCD, optical coupling and scintillator}

An electron multiplying CCD (EM-CCD) is a CCD with an internal gain in the charge domain (before read-out). Because of the internal gain the relative contribution of the read-out noise is reduced significantly, even at high frame rates. The internal gain is achieved by electron multiplication (avalanche multiplication or impact ionization) in the gain register. Details of the EM-CCD technology can be found in Hynecek (2001), Hynecek and Nishiwaki (2003), Robbins and Hadwen (2003) and Plakhotnik et al (2006). The EM-CCD used is the backilluminated CCD 97 from E2V Technologies (figure 1(a)), with $512 \times 512$ active pixels, an active area of $8.192 \times 8.192 \mathrm{~mm}^{2}\left(16 \times 16 \mu \mathrm{m}^{2}\right.$ pixel size $)$ and a quantum efficiency above $90 \%$ for light in the range of 500-650 $\mathrm{nm}$. In order to exchange scintillators easily, the EMCCD is equipped with a $3 \mathrm{~mm}$ straight fiber-optic window. Shown in figure $1(\mathrm{~b})$ is a schematic of the gamma camera. To suppress the thermal dark current noise, the EM-CCD is cooled to $-40{ }^{\circ} \mathrm{C}$ using a Peltier element. The hot side of the Peltier is cooled using a RS44LT cooler purchased from FTS Systems. The cold side of the Peltier element is connected to a copper block, which is in thermal contact with the EM-CCD. For conversion of gamma photons into visible light, continuous $\mathrm{CsI}(\mathrm{Tl})$ scintillators from SCIONIX with a Tl concentration of $0.11 \pm 0.01 \mathrm{~mole} \%$ are used. The detector face of the scintillators is polished using Buehler Micropolish II powder of $1 \mu \mathrm{m}$ particle size. The top and sides of the scintillators were not treated.

The scintillators are optically coupled to the fiber-optic window using Bicron BC-630 silicon optical grease. Due to the critical angle of the fiber-optic window and the scintillatorwindow interface, there is a limited acceptance angle for light on the EM-CCD. The CsI(Tl) scintillators tested have approximately the same area as the EM-CCD active area, the $600 \mu \mathrm{m}$ thick scintillator area is $8.9 \times 10 \mathrm{~mm}^{2}$ and the 1.3 and $2.6 \mathrm{~mm}$ thick scintillator size is $9.2 \times$ $10 \mathrm{~mm}^{2}$. The scintillator thicknesses are $600 \mu \mathrm{m}, 1.3 \mathrm{~mm}$ and $2.6 \mathrm{~mm}$, resulting in an interaction probability for $141 \mathrm{keV}$ gamma photons of $19 \%, 37 \%$ and $61 \%$, respectively.

\subsection{Depth of interaction}

A lack of knowledge about the depth (z-coordinate) within the scintillator at which a scintillation event has occurred (depth of interaction or DOI) causes serious degradation of the detector performance. In pinhole gamma cameras, photons can enter the scintillator at an oblique angle causing the detected image to be blurred due to the varying DOI in the 


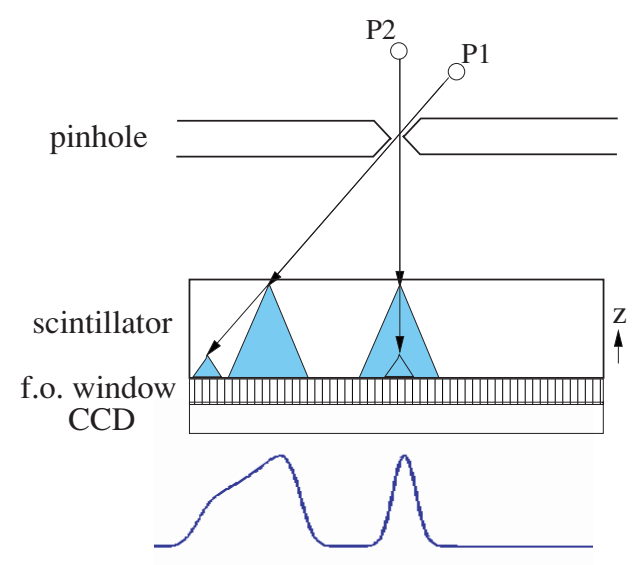

(a)

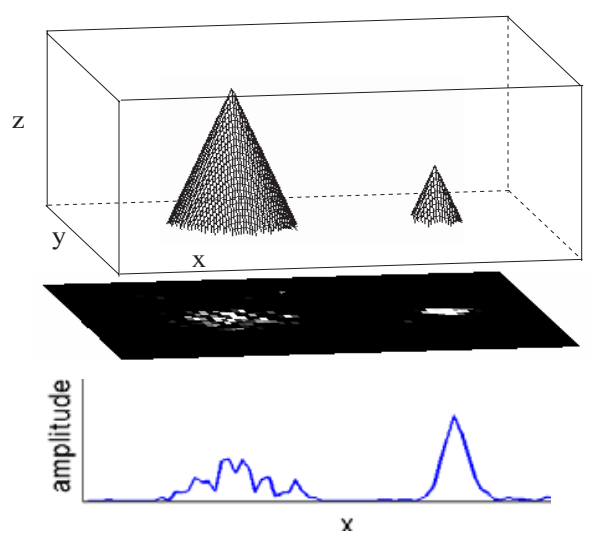

(b)

Figure 2. (a) Diagram of a single pinhole gamma camera with point sources P1 and P2. The gamma photons interact at different depths resulting in a degradation of the spatial resolution (bottom) for photons incident at an oblique angle (DOI effect). Scintillation photons are incident on the entire fiber-optic (fo) window; however, the limited acceptance angle reduces the number of scintillation photons reaching the EM-CCD, resulting in a cone of acceptance. (b) Top: a scintillator with a scintillation with a large light spread $\left(\sigma_{\text {DOI }}\right)$ (left) and a small $\sigma_{\text {DOI }}$ (right). The cones of scintillation light reaching the EM-CCD are shown. Middle: an EM-CCD frame corresponding to the two scintillations. Bottom: a profile of the frame illustrating that the total number of photons (the integrated signal of the scintillation) is independent of $\sigma_{\text {DOI }}$ (or interaction depth).

scintillator (figure 2(a)). This effect in pinhole gamma cameras is called the 'DOI effect' (Hwang et al 2001). This DOI effect will limit the spatial resolution in EM-CCD pinhole cameras, given the scintillator thicknesses required for sufficient interaction probability and the currently attained spatial resolutions.

Apart from the degrading effect on the spatial resolution, the varying DOI can also affect the energy resolution. The scintillation light spread on the EM-CCD depends on the DOI; events further away from the EM-CCD result in a wider light distribution than events occurring closer (figure 2(b)). Therefore, the amplitude of the light spot on the EM-CCD does not provide the full information on the energy of the gamma photon; one must also consider the depth-dependent width of the light distribution. Disregarding the depth-dependence will degrade the energy resolution.

A detection algorithm that can detect the DOI and considers the depth-dependent width can improve the spatial resolution and energy resolution of a gamma camera, and is necessary for high-resolution gamma cameras with sufficient sensitivity.

\subsection{Scintillation detection algorithms}

2.3.1. Gaussian filter algorithm. The GFA is a fast scintillation detection algorithm that can be used for real-time frame analysis (de Vree et al 2005). The CCD frame image $I_{\mathrm{CCD}}$ is corrected for the average dark background by subtracting the average background image $A$ (additionally a threshold can be applied to $I_{\mathrm{CCD}}$ ),

$$
I_{\text {bg.corr. }}=I_{\mathrm{CCD}}-A \text {. }
$$




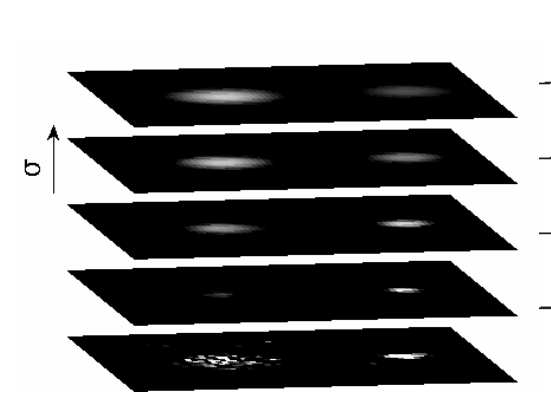

(a)

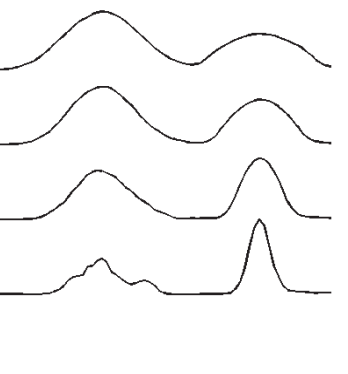

(b)

Figure 3. (a) Four slices of a multi-scale representation of the EM-CCD frame of figure 2(b) (bottom slice), representing two scintillations at different depths. The slices are calculated by convolution of the EM-CCD frame with a Gaussian and multiplication by $\sigma$. (b) Profiles of the multi-scale representation slices. The global maximum of each scintillation in the multi-scale representation (bottom slice for right scintillation, top slice for left scintillation) is found for $\sigma=$ $\sigma_{\text {DOI. }}$

The corrected image $\left(I_{\text {bg.corr. }}\right)$ is then blurred with a 2D Gaussian kernel $(g(x, y, \sigma))$ with fixed width $\sigma$ resulting in a smoothed image:

$$
I_{\text {smoothed }}=I_{\text {bg.corr. }} * g(x, y, \sigma) \text {, }
$$

where $*$ denotes a 2D convolution. To increase speed, this convolution can be implemented as two orthogonal 1D Gaussian convolutions, mathematically identical to a single 2D convolution because of the separability of the Gaussian convolution operation. In this filtered image $\left(I_{\text {smoothed }}\right)$, the scintillations appear as bright regions. Local maxima with amplitudes above a threshold are attributed to scintillation flashes, and their amplitude and position $(x, y)$ are recorded. The GFA does not estimate the DOI or $z$-coordinate of the scintillation and the spatial profile is not corrected for the DOI effect. It is assumed that the energy is proportional to the amplitude, ignoring the depth-dependent light spread.

The width $\sigma$ of $g(x, y, \sigma)$ influences the GFA performance (Heemskerk et al 2007). In this paper, the $\sigma$ of the GFA has been optimized in order to obtain the best possible spatial resolution for each scintillation crystal.

2.3.2. Multi-scale algorithm. The new scintillation detection algorithm presented in this paper, the MSA, is partly inspired by work in computer vision (Koenderink 1984, ter Haar Romeny 1994). The MSA acts as a matched filter that takes the depth-dependent light spread in the continuous scintillator into account and can therefore accurately estimate the DOI.

A single scintillation event will result in a light distribution $I\left(x, y, \sigma_{\mathrm{DOI}}\right)$ on the EM-CCD with a width $\sigma_{\text {DOI }}$ that depends on the DOI (figure 2(b)). The essential step in the MSA is the application of a convolution with a set of Gaussian kernels $g(x, y ; \sigma)$ of increasing width (standard deviation $\sigma$ ) and a subsequent multiplication by the respective standard deviation $\sigma$. This results in the representation of the EM-CCD frame in multiple slices (see figure 3(a)), each slice being characterized by $\sigma$. Scintillation events present in the acquired frame will have a distribution function $P(x, y ; \sigma)=I\left(x, y, \sigma_{\mathrm{DOI}}\right) * g(x, y ; \sigma) \cdot \sigma$ after the convolution step. The global maximum of $P(x, y ; \sigma)$ is in the slice where the width of the Gaussian kernel matches the width of the light distribution on the EM-CCD, i.e. for $\sigma=\sigma_{\text {DOI }}$. Thus $\sigma_{\text {DOI }}$ can be estimated by searching each slice for local maxima and selecting the slice with the global maximum. Once $\sigma$ DOI is determined, the energy and $z$-coordinate of the scintillation are estimated. 
To relate the energy to $\sigma_{\mathrm{DOI}}$ we have to assume a specific light distribution function on the EM-CCD for a single scintillation event. Here it is assumed that all the energy of the gamma photon is deposited at a single interaction position. This assumption is substantiated by the high photoelectric absorption in CsI:Tl (at $141 \mathrm{keV}$ this amounts to $87 \%$ of all inelastic photon interactions (Berger et al 1998)) and the short average range and small energy of fluorescent x-rays (0.1 mm and $35 \mathrm{keV}$, respectively) (Attix 1986). Assuming an isotropic light spread and neglecting optical photon interactions in the scintillator, and with the scintillator surface, the light spread can be approximated by a Gaussian

$$
I\left(x, y, \sigma_{\text {DOI }}\right)=\frac{\varepsilon E}{2 \pi \sigma_{\text {DOI }}^{2}} \mathrm{e}^{-\left(\frac{x^{2}+y^{2}}{2 \sigma_{\text {DOI }}^{2}}\right)},
$$

where $E$ is the energy of the scintillation, $\varepsilon$ is an efficiency factor and the interaction position is at $(x, y)=(0,0)$. The total number of photons on the CCD is proportional to the energy and does not depend on the DOI (illustrated in figure 2(b)), in contrast to the width of the distribution. An explicit evaluation of the convolution results in

$$
P(x, y ; \sigma)=\frac{\varepsilon E \sigma}{2 \pi\left(\sigma_{\text {DOI }}^{2}+\sigma^{2}\right)} \exp \left(-\frac{\left(x^{2}+y^{2}\right)}{2\left(\sigma_{\text {DOI }}^{2}+\sigma^{2}\right)}\right) .
$$

The maximum in the slice characterized by $\sigma$ is given by $P(x=0, y=0 ; \sigma)=\varepsilon E \sigma /$ $2 \pi\left(\sigma_{\text {DOI }}^{2}+\sigma^{2}\right)$. The global maximum (the largest of these maxima) occurs when the width of the Gaussian kernel and the light spread function match, i.e. for $\sigma=\sigma_{\text {DOI }}$. The energy is related to the maximum of $P(x, y ; \sigma)$ by

$$
E=\frac{4 \pi}{\varepsilon} \sigma \max [P(x, y ; \sigma)] \text {. }
$$

The $z$-coordinate of the scintillation can be related to $\sigma_{\mathrm{DOI}}$ with an analytical light spread model or by using experimental data. We have chosen to determine the relation between $\sigma_{\text {DOI }}$ and $z$ by using calibration data of gamma photons incident at a known angle onto the scintillator surface. The calibrated relation between $\sigma_{\mathrm{DOI}}$ and $z$ is used to determine $z$ from the estimated $\sigma_{\text {DOI }}$. During a measurement, the position $(x, y, z)$ and the energy are recorded for every scintillation flash. The knowledge of the $z$-coordinate is used to correct the two-dimensional spatial profile for the DOI effect.

Depending on the desired accuracy and speed, the number of slices can be varied. The range of $\sigma$ depends on the scintillator thickness and has to be such that scintillations at the top of the scintillator (having the largest light spread $\sigma_{\text {DOI }}$ ) can also be detected. To increase speed, the convolutions are implemented as two orthogonal 1D Gaussian convolutions, and the Gaussian kernels are truncated at $2 \sigma$.

\subsection{Measurements}

As a measure of the performance of the MSA, its spatial resolution, energy resolution and signal-to-background ratio are compared with the values obtained by the GFA for CsI(TI) scintillators $0.6,1.3$ and $2.6 \mathrm{~mm}$ in thickness. The number of slices used in the MSA to analyze these measurements is 10 . To determine the spatial resolution, a line pattern from a Tc-99m source (141 keV), projecting through a slit onto the scintillator, is acquired. The spatial resolution is defined as the full width half maximum (FWHM) of the line spread function of the radioactive source, corrected for the width of the gamma photon beam. In our experiments we have investigated both perpendicular incidence (figure 4(a)) and incidence at an angle of $45^{\circ}$ (figure 5). The FWHM energy resolution is obtained by determining the FWHM of the Tc-99m photopeak. 


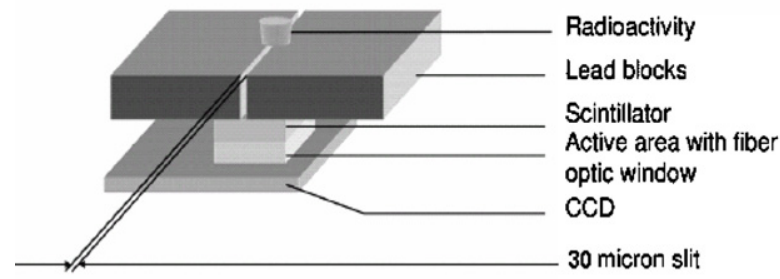

(a)

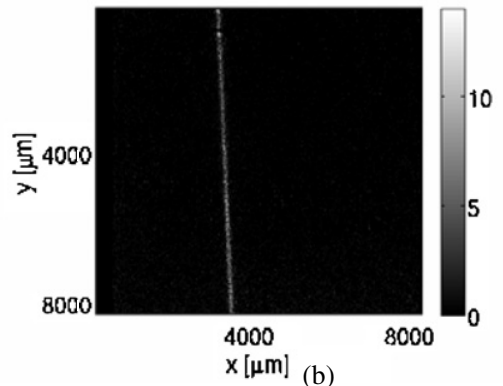

$\mathrm{x}[\mu \mathrm{m}](\mathrm{b})$

Figure 4. (a) Measurement setup for acquiring line pattern images. (b) Image acquired using the GFA of the line pattern of a radioactive Tc-99m source through a $30 \mu \mathrm{m}$ slit for the $600 \mu \mathrm{m}$ CsI(Tl) scintillator.

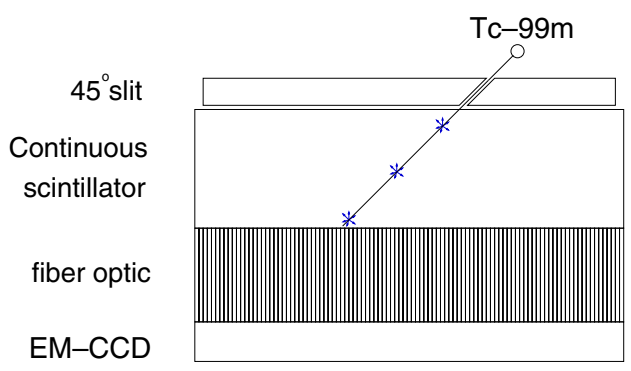

Figure 5. Measurement setup with the $45^{\circ}$ slit.

The signal-to-background ratio (SBR) is defined as the ratio of the number of net signal counts in an irradiated area of the EM-CCD to the number of noise-induced background counts in a non-irradiated area of the same size. The number of net signal counts is obtained by subtracting the number of background counts from the counts in an irradiated area of the same size.

Comparison of the spatial resolution and SBR of the two algorithms is always done for an equal number of net signal counts. This is accomplished by setting an energy window for the MSA and then tuning the detection threshold for the GFA.

\section{Results}

\subsection{Sigma optimization for GFA}

As mentioned in section 2.3.1, the width $\sigma$ of the Gaussian kernel of the GFA is optimized in order to get the best possible GFA performance. The spatial resolutions for the perpendicularly incident gamma photons at different widths $\sigma$ are shown in figure 6 for the $2.6 \mathrm{~mm}$ thick scintillator. For the other thicknesses similar results were obtained. The width $\sigma$ yielding the best spatial resolution is chosen as the optimal $\sigma$ for the comparison with the MSA. This optimal width $\sigma$ increases with scintillator thickness.

\subsection{Spatial resolution}

A line pattern image of a Tc-99m source projected perpendicularly through the $30 \mu \mathrm{m}$ slit and analyzed by the GFA is shown in figure 4(b). For perpendicular incidence, no DOI correction 


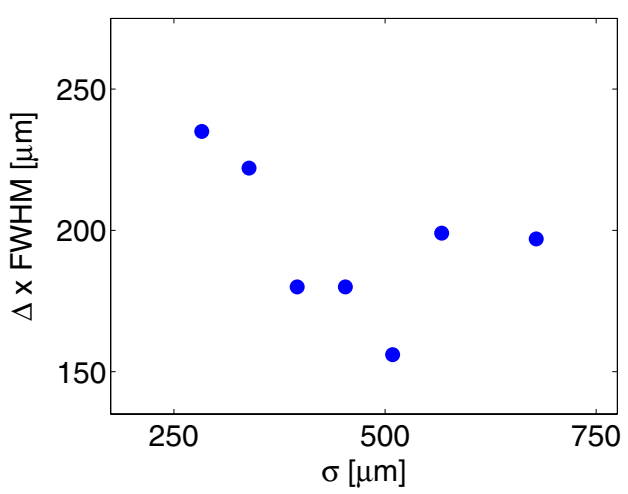

Figure 6. GFA FWHM spatial resolution along the $x$-direction for the $2.6 \mathrm{~mm}$ thick $\mathrm{CsI}(\mathrm{Tl})$ scintillator for different widths $\sigma$ of the Gaussian kernel.

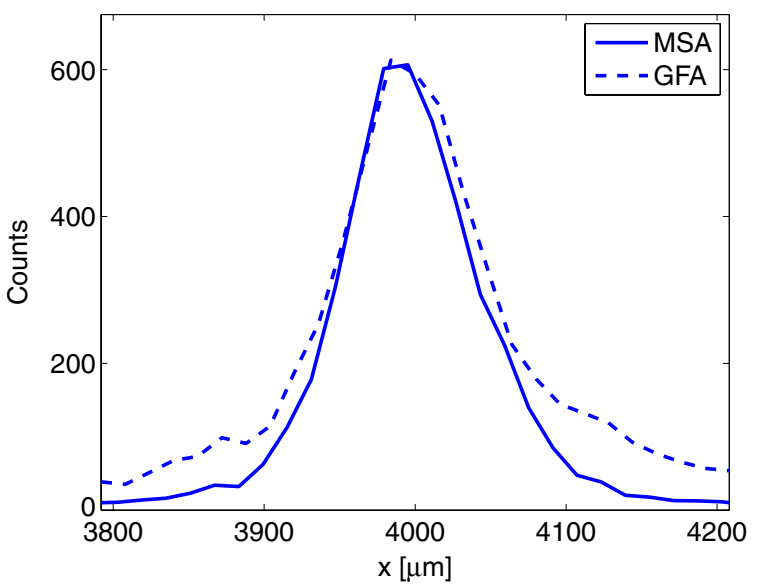

Figure 7. Profiles of the line pattern images for the GFA (dashed line) and MSA (solid line) for a $1.3 \mathrm{~mm}$ thick continuous $\mathrm{CsI}(\mathrm{Tl})$ scintillator.

needs to be applied to the spatial profile. Nevertheless use of the MSA instead of the GFA still slightly improves the spatial resolution by $11 \%, 10 \%$ and $5 \%$ for the scintillator thicknesses of $0.6 \mathrm{~mm}, 1.3 \mathrm{~mm}$ and $2.6 \mathrm{~mm}$, respectively. We believe that this improvement is due to the better match between the kernel width $\sigma$, used to detect the scintillation, and the light spread $\sigma_{\text {DOI }}$, which results in more $\gamma$ photons being detected with a higher accuracy. The slight improvement in the spatial resolution of the line pattern profile for the $1.3 \mathrm{~mm}$ thick scintillator is shown in figure 7. All results are summarized in table 1. The FWHM spatial resolution for both algorithms deteriorates with the scintillator thickness.

When the beam is incident at an oblique angle, an estimation of the $z$-coordinate is necessary to correct the spatial profile for the resolution degrading DOI effect. Figure 8 illustrates the capability of our algorithm to estimate the $z$-coordinate of the interaction for the $1.3 \mathrm{~mm}$ and $2.6 \mathrm{~mm}$ thick scintillators. Density plots are shown in figures $8(\mathrm{a})(1.3 \mathrm{~mm})$ and (c) $(2.6 \mathrm{~mm})$ with the gray scale representing the number of detected scintillation events as a function of the $x$ - and $z$-position, when the gamma photons of the Tc-99m source are incident at an angle of $45^{\circ}$. As expected, the scintillation events are distributed along a line having a slope of approximately $45^{\circ}$ with respect to the scintillator surface plane. Few scintillation 


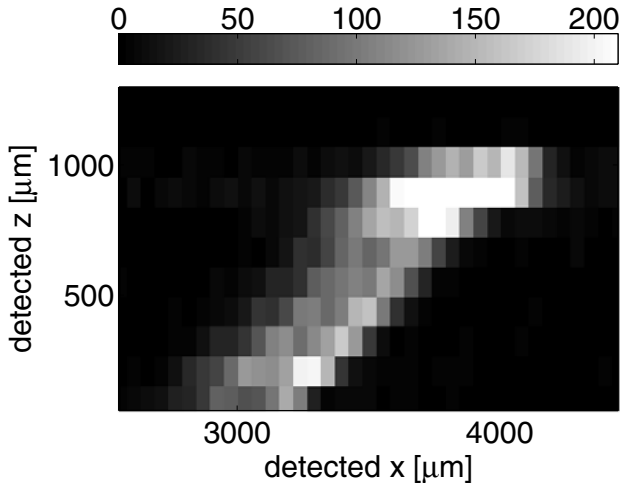

(a)

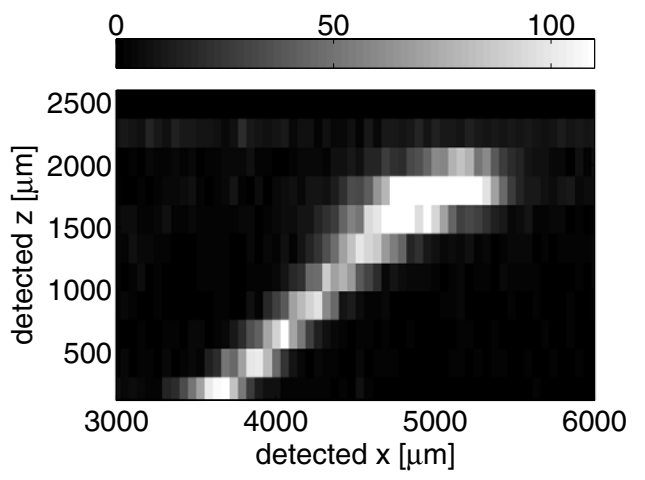

(c)

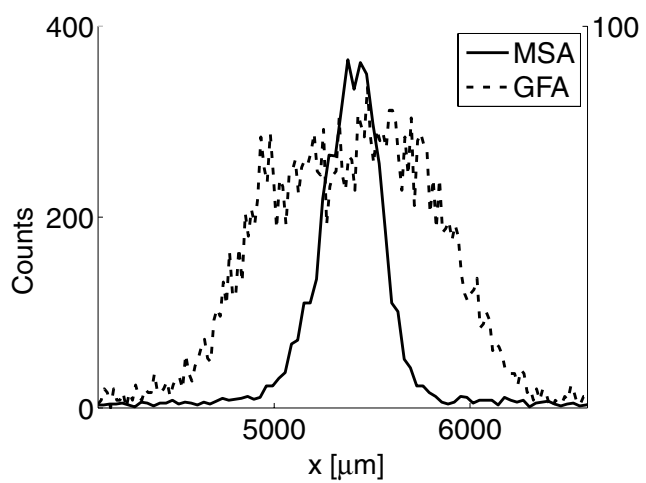

(b)

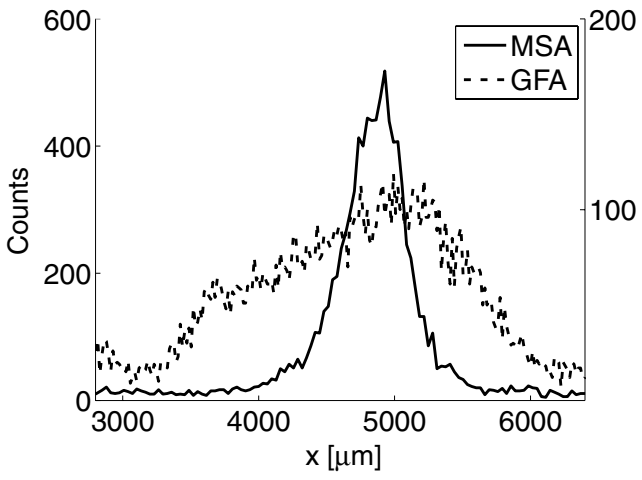

(d)

Figure 8. Density plots of the DOI, $z$, versus position $x$ detected by the MSA are shown on the left. Top: $1.3 \mathrm{~mm}$ thick scintillator; bottom: $2.6 \mathrm{~mm}$ thick scintillator. Image profiles for the GFA (dashed line, left $y$-axis) and DOI-corrected MSA (solid line, right $y$-axis) are shown on the right.

events seem to be present at the top of the scintillator. This small discrepancy is most likely due to mechanical inaccuracies in the setup. The corresponding profiles obtained by the GFA and the MSA with DOI correction are shown in figures 8(b) and (d) and the spatial resolution is listed in table 1. The MSA improves the DOI-degraded spatial resolution by almost a factor of 5 (from $2.0 \mathrm{~mm}$ to $448 \mu \mathrm{m}$ FWHM) for the $2.6 \mathrm{~mm}$ thick scintillator and by actor of 3.3 (from $1.1 \mathrm{~mm}$ to $330 \mu \mathrm{m}$ FWHM) for the $1.3 \mathrm{~mm}$ thick scintillator.

\subsection{Energy resolution and $S B R$}

To investigate the capability of the MSA for improving the energy resolution, we compare the energy spectra obtained with both algorithms for the scintillator thicknesses of $0.6,1.3$ and $2.6 \mathrm{~mm}$ in figure 9. For all scintillator thicknesses, the MSA outperforms the GFA. For the scintillator of $0.6 \mathrm{~mm}$, the GFA does not even show a photopeak, whereas it is clearly visible when the MSA is used. For the $1.3 \mathrm{~mm}$ and $2.6 \mathrm{~mm}$ thick scintillators, the photopeak acquired by the MSA is more narrow than the photopeak obtained by the GFA; respective improvements in the FWHM energy resolution of $34 \%$ and $36 \%$ are obtained. Furthermore, the MSA compared with the GFA results in an energy spectrum with significantly more counts present in the photopeak and less outside the photopeak. As already stated, the results for 


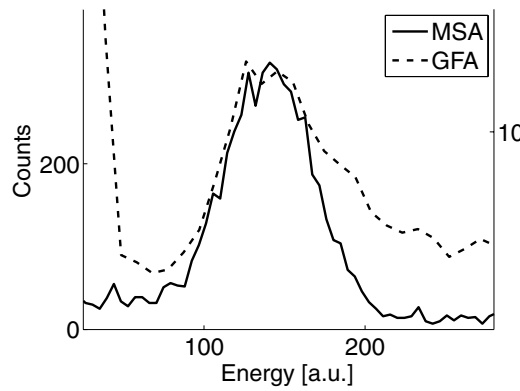

(a)

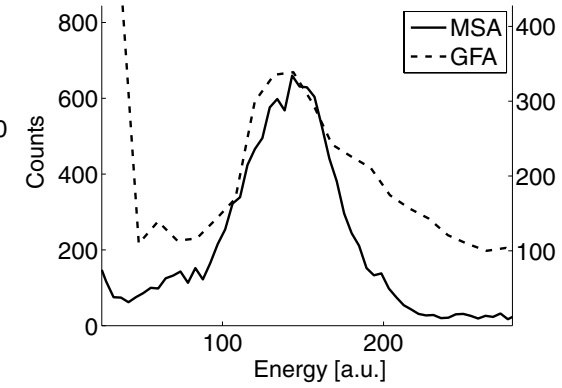

(b)

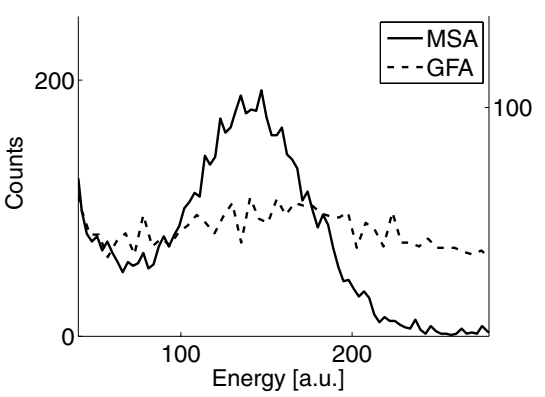

(c)

Figure 9. Energy spectra of the Tc-99m source for the GFA (dotted line, right axis) and MSA (solid line, left axis) for the scintillator thicknesses of $2.6 \mathrm{~mm}$ (a), $1.3 \mathrm{~mm}$ (b) and $600 \mu \mathrm{m}$ (c).

Table 1. Spatial, energy resolution (FWHM) and SBR for the GFA and MSA.

\begin{tabular}{lllll}
\hline & Thickness $(\mathrm{mm})$ & GFA & MSA & Improvement \\
\hline Spatial resolution & 0.6 & $0.45(\mathrm{~mm})$ & $201(\mu \mathrm{m})$ & $2.2 \times$ \\
(oblique angle) & 1.3 & $1.1(\mathrm{~mm})$ & $330(\mu \mathrm{m})$ & $3.3 \times$ \\
& 2.6 & $2.0(\mathrm{~mm})$ & $448(\mu \mathrm{m})$ & $4.5 \times$ \\
Energy resolution & 0.6 & na & $52 \%$ & na \\
& 1.3 & $70 \%$ & $46 \%$ & $34 \%$ \\
SBR & 2.6 & $75 \%$ & $48 \%$ & $36 \%$ \\
& 0.6 & 53 & 70 & $32 \%$ \\
Spatial resolution & 0.6 & 150 & 220 & $47 \%$ \\
(perpendicular) & 1.3 & 195 & 236 & $21 \%$ \\
& 2.6 & $66(\mu \mathrm{m})$ & $59(\mu \mathrm{m})$ & $11 \%$ \\
& & $100(\mu \mathrm{m})$ & $90(\mu \mathrm{m})$ & $10 \%$ \\
\end{tabular}

the MSA were obtained for ten slices (figure 3(a)). The effect of the number of slices on the energy resolution in the MSA has also been investigated and was found to be small.

Compared with the GFA, the MSA improves the SBR by $32 \%, 47 \%$ and $21 \%$ for the scintillators of thicknesses $0.6 \mathrm{~mm}, 1.3 \mathrm{~mm}$ and $2.6 \mathrm{~mm}$, respectively.

\section{Discussion}

In the present work, we have shown that the use of a new analytical scintillation detection algorithm, the MSA, improves the spatial resolution, the energy spectrum and the SBR 
compared to the optimized GFA proposed in de Vree et al (2005). The fast MSA uses information contained in the width of the light spread distribution, allowing an accurate estimation of the energy and a correction to the spatial profile for the DOI effect. The MSA is expected to be significantly faster than a statistical algorithm as this new algorithm does not require the calculation of many iterations.

In this work, we have used a relatively simple model for light spread to facilitate fast blurring operations. More accurate modeling of the light spread function including effects such as inelastic scatter, fluorescent $\mathrm{x}$-rays and reflections on the top and edges of the scintillator can further improve algorithm performance, possibly at the expense of computation time.

In this paper, we did not focus on effects close to the edges of the camera. As in almost any scintillation camera, the spatial resolution is expected to be less good at the edges and could be improved by more advanced modeling. Alternatively, a relative reduction of the number of event detections that suffer from edge effects can be achieved by choosing a larger active area of the gamma camera. A larger active area can be obtained using demagnifying optical tapers (Beekman and de Vree 2005) and larger CCDs, which are currently under development in our department.

We have investigated CsI(Tl) scintillators of different thicknesses. For the thickest scintillator, the improvement in spatial resolution at an oblique angle by the MSA compared with the GFA is most pronounced; an improvement of almost a factor of 5 is reached.

Using the MSA, our gamma camera reaches a spatial resolution of $148 \mu \mathrm{m}$ FWHM with an energy resolution of $46 \%$ FWHM for the $2.6 \mathrm{~mm}$ scintillator (interaction probability $61 \%$ at $141 \mathrm{keV}$ ). While the spatial resolution is much better than the spatial resolution of clinical gamma cameras (typically $3-4 \mathrm{~mm}$ ), the energy resolution is not yet as good. This is not so important for animal imaging, as scatter rejection is often not required due to the lower scatter in animals compared to humans. However, we expect that the use of dense high light output scintillators that are currently under development in our group can improve the energy resolution as well as the spatial resolution and interaction probability.

\section{Conclusions}

In the present work, we have developed a new analytical scintillation detection algorithm, the multi-scale algorithm, and compared it with the Gaussian filter algorithm (de Vree et al 2005). The MSA improves the spatial resolution, the energy spectrum and the SBR compared to the optimized GFA. The MSA can accurately estimate the energy and correct the spatial profile for the DOI effect, improving the resolution up to almost a factor of 5.

The FWHM spatial resolution (both for gamma photons incident perpendicularly and at an oblique angle) obtained by both algorithms deteriorates with the scintillator thickness, due to the increased light spread in thicker scintillators.

Statistical scintillation detection in EM-CCD cameras (Miller et al 2006) may improve the detector performance further; however, long computation times compared to analytical algorithms can be prohibitive in many applications. Besides new scintillators and statistical algorithms, further improvements in spatial resolution, energy resolution and SBR may be obtained by the reduction of noise in the EM-CCD and read-out electronics.

\section{Acknowledgment}

We would like to thank Paul Schotanus of SCIONIX for providing us CsI(Tl) scintillators. 


\section{References}

Attix F H 1986 Introduction to Radiological Physics and Radiation Dosimetry (New York: Wiley) p147

Barber H B 1999 Applications of semiconductor detectors to nuclear medicine Nucl. Instrum. Methods Phys. Res. A 436 102-10

Beekman F J and de Vree G A 2005 Photon-counting versus an integrating CCD-based gamma camera: important consequences for spatial resolution Phys. Med. Biol. 50 N109-19

Beekman F J and Van Der Have F 2007 The pinhole: gateway to ultra-high resolution three-dimensional radio-nuclide imaging Eur. J. Nucl. Med. 34 151-61

Beekman F J, Van Der Have F, Vastenhouw B, Van Der Linden A J A, van Rijk P P, Burbach J P H and Smidt M P 2005 U-SPECT-I: a novel system for submillimeter-resolution tomography with radiolabeled molecules in mice J. Nucl. Med. 46 1194-200

Beekman F J and Vastenhouw B 2004 Design and simulation of a high-resolution stationary SPECT system for small animals Phys. Med. Biol. 49 4579-92

Berger M J, Hubbell J H, Seltzer S M, Chang J, Coursey J S, Sukumar R and Zucker D S 1998 XCOM: photon cross section database http://physics.nist.gov/PhysRefData/Xcom/Text/XCOM.html

de Vree G A, Westra A H, Moody I, van der Have F, Ligtvoet K M and Beekman F J 2005 Photon-counting gamma camera based on an electron-multiplying CCD IEEE Trans. Nucl. Sci. 52 580-8

Heemskerk J W T, Westra A H, Linotte P M, Ligtvoet K M, Zbijewski W and Beekman F J 2007 Front-illuminated versus back-illuminated photon-counting CCD-based gamma camera: important consequences for spatial resolution and energy resolution Phys. Med. Biol. 52 N149-62

Hwang A B, Iwata K and Hasegawa B H 2001 Simulation of depth of interaction effects for pinhole SPECT Nuclear Science Symp. Conf. Record, IEEE vol 3 pp 1293-7

Hynecek J 2001 Impactron-a new solid state image intensifier IEEE Trans. Electron Devices $482238-41$

Hynecek J and Nishiwaki T 2003 Excess noise and other important characteristics of low light level imaging using charge multiplying CCDs IEEE Trans. Electron Devices 50 239-45

Jaszczak R J, Li J Y, Wang H L, Zalutsky M R and Coleman R E 1994 Pinhole collimation for ultra-high-resolution, small-field-of-view SPECT Phys. Med. Biol. 39 425-37

Koenderink J J 1984 The structure of images Biol. Cybern. 50 363-70

Liu Z L, Stevenson G D, Barrett H H, Kastis G A, Bettan M, Furenlid L R, Wilson D W, Woolfenden J M and Pak K Y 2004 Tc-99m glucarate high-resolution imaging of drug sensitive and drug resistant human breast cancer xenografts in SCID mice Nucl. Med. Commun. 25 711-20

Meikle S R, Kench P, Kassiou M and Banati R B 2005 Small animal SPECT and its place in the matrix of molecular imaging technologies Phys. Med. Biol. 50 R45-61

Meng L J 2006 An intensified EMCCD camera for low energy gamma ray imaging applications IEEE Trans. Nucl. Sci. $532376-84$

Miller B W, Barber H B, Barrett H H, Shestakova I, Singh B and Nagarkar V V 2006 Single-photon spatial and energy resolution enhancement of a columnar $\mathrm{CsI}(\mathrm{Tl}) / \mathrm{EMCCD}$ gamma-camera using maximum-likelihood estimation Proc. SPIE 6142 61421T-1

Nagarkar V V, Gupta T K, Miller S R, Klugerman Y, Squillante M R and Entine G 1998 Structured CsI(Tl) scintillators for X-ray imaging applications IEEE Trans. Nucl. Sci. 45 492-6

Nagarkar V V, Shestakova I, Gaysinskiy V, Tipnis S V, Singh B, Barber W, Hasegawa B and Entine G 2006 A CCD-based detector for SPECT IEEE Trans. Nucl. Sci. 53 54-8

Plakhotnik T, Chennu A and Zvyagin A V 2006 Statistics of single-electron signals in electron-multiplying chargecoupled devices IEEE Trans. Electron Devices 53 618-22

Rentmeester M C M, Van Der Have F and Beekman F J 2007 Optimizing multi-pinhole SPECT geometries using an analytical model Phys. Med. Biol. 52 2567-81

Robbins M S and Hadwen B J 2003 The noise performance of electron multiplying charge-coupled devices IEEE Trans. Electron Devices 50 1227-32

Rogulski M M, Barber H B, Barrett H H, Shoemaker R L and Woolfenden J M 1993 Ultra-high-resolution brain SPECT imaging - simulation results IEEE Trans. Nucl. Sci. $401123-9$

Schramm N, Wirrwar A, Sonnenberg F and Halling H 2000 Compact high resolution detector for small animal SPECT IEEE Trans. Nucl. Sci. $471163-7$

ter Haar Romeny B M 1994 Geometry-Driven Diffusion in Computer Division (The Netherlands: Kluwer)

Vastenhouw B, Van Der Have F, Van Der Linden A J A, von Oerthel L, Booij J, Burbach J P H, Smidt M P and Beekman F J 2007 Movies of dopamine transporter occupancy with ultra-high resolution focusing pinhole SPECT Mol. Psychiatry 12 984-7 